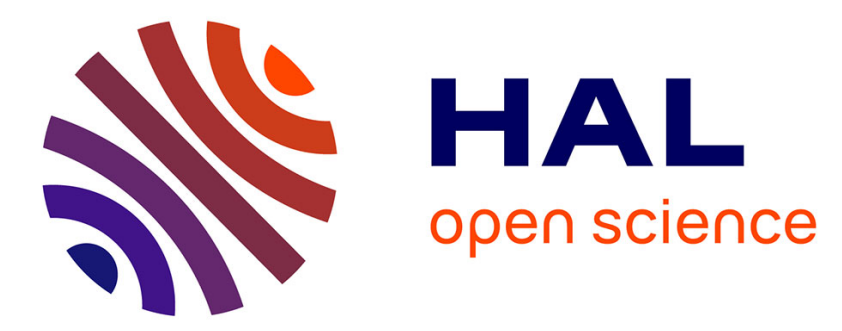

\title{
Relations de Fischer et neutralité de l'inflation
}

Jean-Michel Courtault

\section{To cite this version:}

Jean-Michel Courtault. Relations de Fischer et neutralité de l'inflation. Revue Economique, 1995, 46 (4), pp.1063-1079. halshs-00447850

\section{HAL Id: halshs-00447850 https://shs.hal.science/halshs-00447850}

Submitted on 16 Jan 2010

HAL is a multi-disciplinary open access archive for the deposit and dissemination of scientific research documents, whether they are published or not. The documents may come from teaching and research institutions in France or abroad, or from public or private research centers.
L'archive ouverte pluridisciplinaire HAL, est destinée au dépôt et à la diffusion de documents scientifiques de niveau recherche, publiés ou non, émanant des établissements d'enseignement et de recherche français ou étrangers, des laboratoires publics ou privés. 


\title{
Persée
}

\author{
http://www.persee.fr
}

\section{Relations de Fischer et neutralité de l'inflation.}

\author{
Jean-Michel Courtault \\ Revue économique, Année 1995, Volume 46, Numéro 4 \\ p. $1063-1079$
}

Voir l'article en ligne

\section{Avertissement}

L'éditeur du site «PERSEE » - le Ministère de la jeunesse, de l'éducation nationale et de la recherche, Direction de l'enseignement supérieur, Sous-direction des bibliothèques et de la documentation - détient la propriété intellectuelle et les droits d'exploitation. A ce titre il est titulaire des droits d'auteur et du droit sui generis du producteur de bases de données sur ce site conformément à la loi n`98-536 du 1 er juillet 1998 relative aux bases de données.

Les oeuvres reproduites sur le site «PERSEE » sont protégées par les dispositions générales du Code de la propriété intellectuelle.

Droits et devoirs des utilisateurs

Pour un usage strictement privé, la simple reproduction du contenu de ce site est libre.

Pour un usage scientifique ou pédagogique, à des fins de recherches, d'enseignement ou de communication excluant toute exploitation commerciale, la reproduction et la communication au public du contenu de ce site sont autorisées, sous réserve que celles-ci servent d'illustration, ne soient pas substantielles et ne soient pas expressément limitées (plans ou photographies). La mention Le Ministère de la jeunesse, de l'éducation nationale et de la recherche, Direction de l'enseignement supérieur, Sous-direction des bibliothèques et de la documentation sur chaque reproduction tirée du site est obligatoire ainsi que le nom de la revue et- lorsqu'ils sont indiqués - le nom de l'auteur et la référence du document reproduit.

Toute autre reproduction ou communication au public, intégrale ou substantielle du contenu de ce site, par quelque procédé que ce soit, de l'éditeur original de l'oeuvre, de l'auteur et de ses ayants droit.

La reproduction et l'exploitation des photographies et des plans, y compris à des fins commerciales, doivent être autorisés par l'éditeur du site, Le Ministère de la jeunesse, de l'éducation nationale et de la recherche, Direction de l'enseignement supérieur, Sous-direction des bibliothèques et de la documentation (voir http://www.sup.adc.education.fr/bib/ ). La source et les crédits devront toujours être mentionnés. 


\title{
Relations de Fisher ${ }^{1}$ et neutralité de l'inflation
}

\author{
Jean-Michel Courtault ${ }^{*}$
}

\begin{abstract}
Dans cet article, nous montrons que certaines relations couramment utilisbes en macro-économie monétaire comme la relation de Fisher supposent que les agents économiques sont neutres au risque de richesse nominale. On montre aussi que la relation de Fisher permet de rendre compte de la relation entre inflation et taux d'intérét réel qui est observée dans les économies contemporaines. Toutefois, la relation de Fisher ne peut étre associée à l'hypothèse de neutralité de l'inflation.
\end{abstract}

\section{INTRODUCTION}

Dans cet article, nous allons examiner dans le cadre élémentaire mais complet d'une économie d'échange pur avec un consommateur représentatif la relation de Fisher. Cette relation traduit les liens existant entre le taux d'intérêt nominal, le taux d'intérêt réel et le taux d'inflation anticipé.

Nous montrerons, dans un premier temps, que l'on peut obtenir plusieurs relations couramment utilisées en macro-économie monétaire comme la parité des taux d'intérêt couverts, la relation de Fisher et l'hypothèse d'efficience du marché des changes à partir d'un raisonnement par arbitrage. On sait, en effet, que sous l'hypothèse d'absence d'opportunités d'arbitrage le prix de chaque actif peut être exprimé comme une fonction linéaire des prix des états de la nature, mais que ceux-ci ne sont pas uniques, sauf dans le cas des marchés complets. On sait aussi que ces prix peuvent être réinterprétés, à un coefficient d'homogénéité près, comme les probabilités subjectives d'un agent neutre à l'égard du risque. Il est alors facile d'établir, en utilisant ces probabilités corrigées du risque pour calculer les anticipations, que la relation de Fisher et l'hypothèse d'efficience du marché des changes sont des relations vraies quel que soit le comportement vis-à-vis du risque des agents.

* CRESE, Faculté de droit Université de Franche-Comté, avenue de l'Observatoire, 25030 Besançon.

1. Je remercie J.-P. Laffargue, M. Mougeot ainsi qu'un rapporteur anonyme de la Revue économique pour leurs commentaires sur une version préliminaire de cet article. Je remercie également $B$. Solnik. 
Puis nous montrerons que, lorsqu" on considère la vraie distribution des probabilités, il est possible d'obtenir deux formes différentes pour la relation de Fisher suivant qu'on suppose que l'agent représentatif est neutre au risque de richesse nominale ou neutre au risque de consommation. La forme traditionnelle de la relation de Fisher est obtenue lorsque l'agent représentatif est neutre au risque de richesse nominale.

Enfin, nous étudierons si la relation de Fisher possède les propriétés de neutralité qui lui sont couramment attribuées. En particulier, est-ce que la relation de Fisher garantit ou non la neutralité de l'inflation ? En d'autres termes, est-ce qu'une augmentation du taux d'inflation anticipé se traduit par une augmentation du taux d'intérêt nominal de façon à maintenir constant le taux d'intérêt réel ? La question n'est pas triviale puisque la relation de Fisher ne nous indique pas les variables qui doivent s'ajuster, elle indique seulement que le taux d'intérêt réel et le taux d'intérêt nominal doivent s'ajuster proportionnellement à l'inflation (on a, en effet, une seule équation pour deux variables endogènes). Seul un modèle d'équilibre général peut nous permettre de répondre de façon définitive à cette question.

Ainsi nous montrerons que la relation de Fisher n'implique pas, contrairement à ce que l'on croit, la neutralité de l'inflation' ${ }^{1}$. En effet, l'étude de statique comparative du modèle développé dans la section 4 nous montrera qu'une augmentation de l'inflation anticipée a pour conséquence soit de diminuer le taux d'intérêt réel, dans le cas où les agents sont neutres au risque de richesse nominale, soit d'augmenter de façon moins que proportionnelle le taux d'intérêt nominal si les agents sont neutres au risque de consommation. Nous verrons cependant que la relation de Fisher sous sa forme traditionnelle permet de rendre compte de certains faits stylisés comme la corrélation négative entre le taux d'intérêt réel et l'inflation.

Le modèle que nous étudierons a été, en particulier ces dernières années, abondamment utilisé sous des formes diverses ${ }^{2}$. Nous considérons un consommateur représentatif ne vivant que deux périodes. En première période, il consomme et épargne et, en seconde période, il dépense toute sa richesse accumulée. Son objectif étant la maximisation de l'espérance de l'utilité de la consommation présente et future, on déduit des conditions du premier ordre associées à la maximisation de son objectif lorsque tous les marchés sont à l'équilibre des relations sur les prix des biens et des actifs financiers. En modifiant l'ensemble des actifs à partir duquel le consommateur compose son portefeuille, on obtient des relations différentes. Ainsi, dans la section 3, obtiendrons-nous la relation entre taux d'intérêt réel et nominal en supposant

1. Habituellement, on montre, comme par exemple I. Hansson et C. Stuart [1986], que l'inflation n'est pas neutre, quoique la relation de Fisher soit respectée, à cause des distorsions entraînées par la fiscalité.

2. On peut trouver une revue récente de la littérature dans T. Sargent [1987]. Le modèle que nous utiliserons est cependant plus proche de celui que l'on peut trouver dans R. Roll et B. Solnik [1979] et surtout dans J. Frenkel et A. Razin [1980]. Notre travail reprend, en partie, celui qui a été fait dans les deux articles précédents ; cependant, nous portons plus particulièrement l'attention sur plusieurs points importants qui, à notre connaissance, ont été insuffisamment soulignés. Nous étudions de plus les propriétés de statique comparative des modèles utilisés. 
que l'agent représentatif peut investir son épargne dans un actif à rendement réel constant et dans un actif à rendement nominal constant. Nous discuterons en conclusion de l'intérêt ainsi que des limites des modèles décrits ci-dessus.

\section{RELATIONS D'ARBITRAGE EN MACRO-ÉCONOMIE}

On dit qu'il existe des opportunités d'arbitrage s'il est possible de gagner de l'argent sans risque et sans mise de fonds initiale. Cela signifie dans le cadre d'une économie d'échange à deux périodes qu'il est possible de former un portefeuille $n$ parmi $\mathbf{N}$ actifs financiers sans coût (en vendant à découvert certains actifs ou en empruntant) et dont le paiement est positif dans les $S$ états de la nature (le rendement des actifs que l'on a pu acheter grâce à la vente à découvert est ainsi suffisant pour permettre le remboursement des emprunts). En notant $Y$ la matrice $(\mathrm{S} \times \mathrm{N})$ des paiements des actifs et $p$ le vecteur des prix des actifs l'existence d'opportunités d'arbitrage s'écrit :

$$
\exists n / \mathrm{Y} n \geq 0 \text { et } p^{\dagger} n \leq 0
$$

avec au moins une inégalité stricte. Pour qu'il n'existe pas d'opportunités d'arbitrage, il faut et il suffit qu'il existe un vecteur de prix d'états $q>0$ tel que l'on $\mathbf{a}^{1}$ :

$$
\mathrm{Y}^{\prime} q=p
$$

Le prix d'état $q_{s}$ s'interprète comme le prix qu'il faudrait verser en l'absence d'opportunités d'arbitrage pour obtenir un franc dans l'état de la nature $s$. Comme l'actif $i$ rapporte $y_{i s}$ dans l'état $s$ il est normal qu'il coûte :

$$
p_{i}=\sum_{s=1}^{\mathrm{s}} q_{s} y_{i s}
$$

On comprend alors que si cette égalité n'était pas observée ou bien si certains prix d'état était nul ou même négatif, il serait possible de tirer parti de ces opportunités pour réaliser sans risque et sans mise de fonds initiale des profits infinis.

Supposons maintenant qu'il existe un actif à rendement nominal certain, un actif à rendement réel certain, un actif à rendement nominal certain en monnaie étrangère, un contrat de change à terme. La matrice des paiements nominaux $\mathbf{Y}$ exprimés en monnaie nationale s'écrit :

$$
\mathbf{Y}=\left(\begin{array}{cccc}
(1+\mathrm{R}) & (1+r) \mathrm{P}_{21} / \mathrm{P}_{1} & \left(1+\mathrm{R}^{*}\right) e_{21} & \left(e_{21}-\mathrm{F}\right) \\
\vdots & \vdots & \vdots & \vdots \\
(1+\mathrm{R}) & (1+r) \mathrm{P}_{2 \mathrm{~S}} / \mathrm{P}_{1} & \left(1+\mathrm{R}^{*}\right) e_{2 \mathrm{~S}}\left(e_{2 \mathrm{~S}}-\mathrm{F}\right)
\end{array}\right)
$$

ou $\mathrm{R}\left(\mathrm{R}^{*}\right)$ est le taux de rendement net de l'actif sans risque exprimé en monnaie nationale (étrangère), $r$ le taux d'intérêt réel, $\mathrm{P}_{1}$ le niveau général des prix

\footnotetext{
1. Cf. H. Varian [1988].
} 
en première période, $\mathrm{P}_{2 s}$ le niveau général des prix en deuxième période dans l'état $s, e_{2 s}$ est le taux de change au comptant d'une unité de monnaie étrangère exprimé en monnaie nationale en deuxième période et dans l'état $s$ et où $F$ est le taux de change à terme d'une unité de monnaie étrangère.

Le paiement réel d'un actif étant égal par définition à son paiement nominal en monnaie nationale divisé par le taux d'inflation brut $\left(\mathbf{P}_{2} \sqrt{\mathbf{P}_{1}}\right)$ il s'ensuit que le paiement réel du deuxième actif est égal à $(1+r)$ dans tous les états de la nature et donc est certain. L'obligation étrangère, sans risque en termes de la monnaie étrangère, rapporte $\left(1+R^{*}\right) e_{2 s}$ unités de monnaie nationale. L'achat d'une unité de monnaie étrangère coûtera à terme $\mathrm{F}$ alors qu'elle vaut $e_{2 s}$ sur le marché des changes, le bénéfice est donc égal à la différence $\left(e_{2 s}-\mathrm{F}\right)$.

Le prix de chacun de ces actifs est donné par le vecteur :

$$
p^{\prime}=\left(1,1, e_{1}, 0\right)
$$

où $e_{1}$ est le taux de change au comptant d'une unité de monnaie étrangère en première période et où nous supposons qu'il n'existe pas de marge de couverture pour le contrat à terme.

En l'absence d'opportunité d'arbitrage, il existe d'après (2) un vecteur de prix d'états $q$ strictement positifs tels que ${ }^{1}$ :

$$
\left\{\begin{array}{l}
\sum_{s=1}^{S} q_{s}(1+\mathrm{R})=1 \\
\sum_{s=1}^{\mathrm{S}} q_{s}(1+r) \mathrm{P}_{2 s} / \mathrm{P}_{1}=1 \\
\sum_{s=1}^{\mathrm{S}} q_{s}\left(1+\mathrm{R}^{*}\right) e_{2 s}=e_{1} \\
\sum_{s=1}^{S} q_{s}\left(e_{2 s}-\mathrm{F}\right)=0
\end{array}\right.
$$

On déduit de la première de ces relations que le rendement brut de l'actif sans risque en terme nominal est égal à l'inverse de la somme des prix d'états

$$
(1+\mathrm{R})=\left(\sum_{s=1}^{s} q_{s}\right)^{-1}
$$

En effet, la somme des prix d'états correspond à la valeur actualisée de 1 franc perçu dans tous les états de la nature : c'est donc le prix de l'actif sans risque. L'inverse de cette somme est égale au rendement brut d'une obligation à coupon zéro rapportant 1 franc avec certitude. Réciproquement, $(1+R)^{-1}$ est le prix d'une telle obligation.

1. Notons que les relations du système (5) ne se trouveraient pas modifiées par la prise en compte d'autres actifs dans la mesure où ils ne créent pas d'opportunités d'arbitrage. 
De la seconde relation, on déduit que le prix, en termes réels, d'une obligation à coupon zéro rapportant une unité de bien de consommation avec certitude est égal à la somme des prix d'états pondérés par le taux brut de l'inflation :

$$
(1+r)^{-1}=\sum_{s=1}^{s} q_{s} \mathrm{P}_{2 s} / \mathrm{P}_{1}
$$

En effet, un tel actif rapporte en terme nominal le prix futur du bien de consommation. En l'absence d'opportunités d'arbitrage, le prix nominal de cet actif $\left(\mathrm{P}_{1}(1+r)^{-1}\right)$ doit être égal à la valeur actualisée de son paiement nominal.

De la troisième relation, on déduit que le prix en monnaie étrangère d'une obligation étrangère à coupon zéro rapportant une unité de monnaie étrangère avec certitude est égal à la somme des prix d'état pondérés par le taux brut de la dépréciation de la monnaie nationale en termes de la monnaie étrangère :

$$
\left(1+\mathrm{R}^{*}\right)^{-1}=\sum_{s=1}^{s} q_{s} e_{2 s} / e_{1}
$$

En effet, une unité de monnaie étrangère vaudra $\tilde{e}_{2}$ francs. En l'absence d'opportunités d'arbitrage, le prix en monnaie nationale de cette obligation étrangère $\left(e_{1}\left(1+\mathrm{R}^{*}\right)^{-1}\right)$ doit être égal à la valeur actualisée de son paiement.

De la dernière relation, on déduit que le taux de change à terme est égal au rapport de la valeur actualisée du taux de change futur au comptant divisé par le prix d'une obligation rapportant 1 franc avec certitude :

$$
\mathrm{F}=\frac{\sum_{s=1}^{s} q_{s} e_{2 s}}{\sum_{s=1}^{s} q_{s}}
$$

En effet, on peut se procurer une unité de monnaie étrangère en deuxième période soit sur le marché des changes à terme à un prix $F$ soit sur le marché des changes au comptant à un prix $\tilde{e}_{2}$. En l'absence d'opportunités d'arbitrage, ces deux contrats doivent avoir la même valeur présente ${ }^{1}$.

En remplaçant dans l'équation (9) le numérateur par sa valeur que l'on obtient à partír de (8) et le dénominateur par l'équation (6), on obtient la parité des taux d'interêts couverts :

$$
\frac{\left(1+\mathrm{R}^{*}\right)}{(1+\mathrm{R})}=e_{1} / \mathrm{F}
$$

On peut également obtenir cette relation par le raisonnement d'arbitrage suivant. On peut se procurer une unité monétaire étrangère en deuxième période de

1. La valeur actualisée d'un paiement de $\mathrm{F}$ francs avec certitude est égale à $\sum_{s=1}^{S} q_{s} \mathrm{~F}$ et la valeur actualiśé d'un paiement aléatoire de $\tilde{e}_{2}$ francs est égale à $\sum_{s=1}^{S} q_{s} e_{2 s}$; d'où la relation (9). 
deux façons. Soit par le marché à terme, ce qui coutera F. Soit en empruntant une unité monétaire nationale que l'on placera sur le marché obligataire étranger. Le cout de chaque unité monétaire étrangère que l'on se sera procuré de cette manière se monte à $(1+\mathrm{R}) \div\left(1 / e_{1}\right)\left(1+\mathrm{R}^{*}\right)$. La relation $(10)$ nous dit que ces deux types d'opérations doivent être aussi coûteux l'un que l'autre.

En multipliant chaque membre de l'équation (6) par les membres correspondant de l'équation (7), on obtient la relation suivante :

$$
\left(\frac{1+\mathrm{R}}{1+r}\right)=\frac{\sum_{s=1}^{\mathrm{S}} q_{s} \mathrm{P}_{2 s}}{\mathrm{P}_{1} \sum_{s=1}^{S} q_{s}}
$$

En posant $\hat{\pi}_{s}=q_{s} /\left(\sum_{s=1}^{s} q_{s}\right)$, on peut réccrire cette demière équation pour obtenir la relation de Fisher :

$$
\frac{(1+\mathrm{R})}{(1+r)}=\sum_{s=1}^{\mathrm{S}} \hat{\pi}_{s} \mathrm{P}_{2 s} / \mathrm{P}_{1}=\hat{\mathrm{E}}(1+\tilde{i})
$$

où $\tilde{i}$ est le taux d'inflation net et où $\hat{E}$ est l'espérance mathématique relativement à la distribution de probabilité $\hat{\pi}_{s}$. En effet, $\hat{\pi}_{s}$ peut être considéré comme une probabilite puisque :

$$
0<\hat{\pi}_{s}<1 \text { et } \sum_{s=1}^{S} \hat{\pi}_{s}=1
$$

On appelle $\hat{\pi}_{s}$ les probabilités corrigées du risque car, pour cette distribution de probabilité, le risque d'un actif n'est pas compensé par un paiement élevé.

Tout se passe comme si les agents étaient neutres au risque ${ }^{1}$.

De même, on peut réécrire (9) pour obtenir la relation d'efficience du marché des changes :

$$
\sum_{s=1}^{s} \hat{\pi}_{s} e_{2 s}=\hat{\mathrm{E}} e_{2}
$$

On pourrait obtenir d'une façon analogue dans le cadre dynamique d'une économie à plusieurs périodes la relation sur la structure par terme des taux d'intérêt.

Les relations (11) et (12) sont la généralisation des relations connues sous le nom de relation de Fisher et de relation d'efficience du marché des changes. Elles montrent qu'elles sont obtenues pour des distributions de probabilités quelconques lorsque les agents sont neutres au risque. L'objet des sections suivantes est de montrer à quel type de neutralité au risque elles correspondent. De plus, nous chercherons à établir l'influence de modifications dans la distribution du taux d'inflation sur les taux d'intérêt réel et nominal.

1. Cf. G. Demange et J.-C. Rochet [1992], Corollaire 6, p. 62. 
L'inconvénient de l'approche précédente tient à ce qu'il existe dans le cas général des marchés incomplets une infinité de prix d'états ainsi qu'une infinité de distribution de probabilités compatibles avec l'équation (2). Il n'est donc pas possible d'obtenir des résultats significatifs sur la base d'exercices de statique comparative à partir du système d'équations (5). En effet, suivant le vecteur des prix d'états choisis, une augmentation de l'inflation anticipée pourrait tout aussi bien augmenter que diminuer le taux d'intérêt nominal. Nous verrons cependant que, lorsqu'il est possible de représenter l'économie par un consommateur représentatif, c'est le cas notamment lorsque l'économie est optimale au sens de Pareto $^{1}$, un vecteur particulier de prix d'états et de probabilités corrigées du risque s'impose. Ce sont les vecteurs que l'on obtient à partir des conditions nécessaires d'optimalité du premier ordre du programme du consommateur représentatif. En effet, dans une économie optimale au sens de Pareto, les taux marginaux de substitution sont égaux pour tous les consommateurs et, par definition, ces taux marginaux de substitution sont égaux aux prix d'états. On peut alors déterminer les conséquences d'une augmentation du taux d'inflation ou de son incertitude sur les taux d'intérêt réel et nominal en examinant les conséquences de ces variations sur les taux marginaux de substitution et en utilisant les formules d'évaluation par arbitrage (6) et (7).

\section{RELATIONS DE FISHER ET NEUTRALITÉ AUX RISQUES}

Considérons un individu représentatif pouvant affecter son épargne sous la forme d'un actif $(b)$ à rendement réel $(r)$ constant et d'un actif (B) à rendement nominal (R) constant. Son objectif étant la maximisation de l'espérance de l'utilité, on formalise son comportement de la façon suivante :

$$
\begin{array}{cc}
\underset{\mathrm{C}_{1}, \mathrm{~B}, b}{\mathrm{Max}} & \mathrm{U}\left(\mathrm{C}_{1}\right)+\beta \sum_{s=1}^{\mathrm{S}} \pi_{s} \mathrm{U}\left(\mathrm{C}_{2 s}\right) \\
& \mathrm{P}_{1} \mathrm{C}_{1}+\mathrm{B}+b=\mathrm{W}_{1}
\end{array}
$$

où $P_{1}$ est le prix de la consommation en première période $\left(C_{1}\right), C_{2 s}$ la consommation en seconde période dans l'état $s=1, \ldots, \mathrm{S}$ et $\mathrm{W}_{1}$ est la richesse initiale. On suppose que l'individu ne vit que deux périodes et qu'il consomme toute sa richesse finale

$$
\mathrm{P}_{2 s} \mathrm{C}_{2 s}=(1+\mathrm{RB})+(1+r) b \mathrm{P}_{2 s} / \mathrm{P}_{1} \quad s=1, \ldots, \mathrm{S}
$$

où $\mathrm{P}_{2 s}$ est le prix de la consommation future dans l'état $s$. Notons que c'est l'élément fondamental d'incertitude dans ce modèle : l'incertitude quant à la consommation future en résulte.

1. Cf. G. Demange et J.-C. Rochet [1992], proposition 8, p. 94-96. 
On déduit du Lagrangien associé au problème de maximisation les conditions du premier ordre suivantes :

$$
\begin{gathered}
\mathrm{U}^{\prime}\left(\mathrm{C}_{1}\right) / \mathrm{P}_{1}=\lambda \\
\beta(1+\mathrm{R}) \sum_{s=1}^{\mathrm{S}} \pi_{s} \mathrm{U}^{\prime}\left(\mathrm{C}_{2 s}\right) / \mathrm{P}_{2 s}=\lambda \\
\beta(1+r) \sum_{s=1}^{\mathrm{S}} \pi_{s} \mathrm{U}^{\prime}\left(\mathrm{C}_{2 s}\right)=\lambda \mathrm{P}_{1} \\
\mathrm{P}_{1} \mathrm{C}_{1}+\mathrm{B}+b=\mathrm{W}_{1}
\end{gathered}
$$

où $\lambda$ est le multiplicateur de Lagrange associé à la contrainte budgétaire (17). On peut montrer, grâce au théoreme de l'enveloppe ${ }^{1}$, que c'est aussi l'utilité marginale de la richesse en première période. Les équations (14)-(16) révèlent le principe sur lequel se fonde le comportement de consommation et d'investissement d'un agent rationel en situation d'incertitude ${ }^{2}$.

D'après (14), un agent rationnel doit pousser la dépense de consommation jusqu'au point où l'utilité marginale qu'il en retire, soit $\left(1 / \mathbf{P}_{1}\right) \times \mathrm{U}^{\prime}\left(\mathrm{C}_{1}\right)$, est égale à l'utilité marginale de la richesse nominale $\lambda$ à laquelle il renonce par sa dépense. La dépense de un franc supplémentaire permet, en effet, à l'agent d'acheter $1 / \mathbf{P}_{1}$ unités de bien de consommation qui lui procurent chacune approximativement $U^{\prime}\left(C_{1}\right)$ unités d'utilité. Ou encore, considérant que $\lambda P_{1}$ est l'utilité marginale de la richesse réelle, l'agent doit consommer jusqu'au point où l'utilité marginale de la consommation est égale à l'utilité marginale de la richesse réelle. En effet, une unité du bien de consommation (qui est l'unité en termes de laquelle la richesse réelle est mesurée) vaut $P_{1}$ francs et chaque franc procure (à la limite) $\lambda$ unités d'utilité.

Avec l'épargne ainsi formée, il doit investir dans un actif à rendement nominal (réel) certain jusqu'au point où l'espérance mathématique actualisée de l'utilité marginale de la richesse nominale (consommation) future soit égale à l'utilité marginale de la richesse nominale (consommation) présente. $^{3}$

On peut réécrire le système formé des équations (14)-(16) de la manière suivante :

$$
\begin{aligned}
& \sum_{s=1}^{S}\left[\beta \pi_{s} \frac{\mathrm{U}^{\prime}\left(\mathrm{C}_{2 s}\right) / \mathrm{P}_{2 s}}{\mathrm{U}^{\prime}\left(\mathrm{C}_{1}\right) / \mathrm{P}_{1}}\right](1+\mathrm{R})=1 \\
& \sum_{s=1}^{s}\left[\beta \pi_{s} \frac{\mathrm{U}^{\prime}\left(\mathrm{C}_{2 s}\right) / \mathrm{P}_{2 s}}{\mathrm{U}^{\prime}\left(\mathrm{C}_{1}\right) / \mathrm{P}_{1}}\right](1+r) \frac{\mathrm{P}_{2 s}}{\mathrm{P}_{1}}=1
\end{aligned}
$$

1. Cf. H. Varian [1984], p. 327-329.

2. À notre connaissance, ce principe a été énoncé pour la première fois par $P$. Massé [1948].

3. Cf. (15) et (16); $\beta$ est le coefficient d'actualisation et $U^{\prime}\left(C_{2 s}\right) / P_{2 s}$ est l'utilité marginale future de la richesse nominale dans l'état $s$. 
On peut donc prendre comme prix d'états et comme probabilités corrigées du risque les expressions suivantes :

$$
\begin{gathered}
q_{s}=\beta \pi_{s} \frac{\mathrm{U}^{\prime}\left(\mathrm{C}_{2 s}\right) / \mathrm{P}_{2 s}}{\mathrm{U}^{\prime}\left(\mathrm{C}_{1}\right) / \mathrm{P}_{1}} \\
\hat{\pi}_{s}=\beta \pi_{s} \frac{\pi_{s} \mathrm{U}^{\prime}\left(\mathrm{C}_{2 s}\right) / \mathrm{P}_{2 s}}{\sum_{s=1}^{\mathrm{S}} \pi_{s} \mathrm{U}^{\prime}\left(\mathrm{C}_{2 s}\right) / \mathrm{P}_{2 s}}
\end{gathered}
$$

puisque $q_{s}$ est bien une solution strictement positive de (2). Les prix d'états $q_{s}$ sont égaux aux taux marginaux de substitution de la richesse nominale présente à la richesse nominale dans l'état $s$. Les prix d'états sont en effet les prix qu'il faut payer en première période pour obtenir un franc dans l'état $s$. Les taux marginaux de substitution sont égaux par définition à la quantité de monnaie que l'on doit abandonner en première période pour percevoir en échange un franc dans l'état $s$ de façon à maintenir l'utilité constante. L'équation (20) nous indique qu'à l'équilibre ces deux grandeurs doivent être égales. En normalisant l'espérance de l'utilité marginale de la richesse nominale, l'équation (21) nous indique la façon dont on peut obtenir les probabilités corrigées du risque. Il suffit, en effet, de multiplier les vraies probabilités par l'utilité marginale de la richesse nominale dans le même état de la nature.

Le système formé des $S+4$ équations (13)-(17) permet de déterminer les $S+4$ inconnues $\left(C_{2 s}, \lambda, P_{1}, r, R\right)$ lorsque les marchés du bien de consommation et des actifs financiers sont en équilibre ${ }^{1}$. On peut en déduire la relation suivante entre taux d'intérêt réel et nominal :

$$
\frac{(1+\mathrm{R})}{(1+r)}=\frac{\sum_{s=1}^{s} \pi_{s} \mathrm{U}^{\prime}\left(\mathrm{C}_{2 s}\right) / \mathrm{P}_{1}}{\sum_{s=1}^{\mathrm{S}} \pi_{s} \mathrm{U}^{\prime}\left(\mathrm{C}_{2 s}\right) / \mathrm{P}_{2 s}}
$$

que l'on peut récrire de deux façons différentes ${ }^{2}$ :

$$
\begin{aligned}
& \frac{(1+\mathrm{R})}{(1+r)}=\frac{\mathrm{E} \tilde{\mathrm{P}}_{2}}{\mathbf{P}_{1}}+\frac{\operatorname{Cov}\left(\mathrm{U}^{\prime}\left(\tilde{\mathrm{C}}_{2}\right) / \tilde{\mathbf{P}}_{2}, \tilde{\mathbf{P}}_{2} / \mathrm{P}_{1}\right)}{\mathrm{E}\left(\mathrm{U}^{\prime}\left(\tilde{\mathrm{C}}_{2}\right) / \tilde{\mathbf{P}}_{2}\right)} \\
& \frac{(1+r)}{(1+\mathrm{R})}=\frac{\mathrm{E} \tilde{\delta}_{2}}{\tilde{\delta}_{1}}+\frac{\operatorname{Cov}\left(\mathrm{U}^{\prime}\left(\tilde{\mathbf{C}}_{2}\right), \tilde{\delta}_{2} / \tilde{\delta}_{1}\right)}{\mathrm{E}\left(\mathrm{U}^{\prime}\left(\tilde{\mathrm{C}}_{2}\right)\right)}
\end{aligned}
$$

1. En imposant au marché de la consommation future d'être en équilibre les prix $P_{2 s}$ seraient déterminés par le modele. Cependant, en les considérant comme exogènes au modèle, nous pourrons étudier l'influence de l'inflation sur les taux d'intérêt.

2. En utilisant la relation $E(\tilde{X} \tilde{Y})=\operatorname{Cov}(\tilde{X}, \tilde{Y})+E(\tilde{X}) E(\tilde{Y})$, où $\tilde{X}$ et $\tilde{Y}$ sont des variables aléatoires et où $\mathrm{E}($ ) et $\operatorname{Cov}()$ sont l'espérance mathématique et la covariance, respectivement. 
où $\delta_{i}$ est le pouvoir d'achat d'une unité monétaire en $i^{\text {ème }}$ période. Les relations (23) et (24) définissent respectivement un taux d'inflation et un taux d'appréciation de l'unité monétaire implicite. Le taux d'inflation (d'appréciation monétaire) implicite, défini par le rapport du taux d'intérêt nominal au taux d'intérêt réel (ou l'inverse de ce rapport), est égal au taux d'inflation (d'appréciation) anticipé dans le cas de certitude subjective ou dans le cas de constance de l'utilité marginale de la richesse nominale (consommation). Dans les autres cas, il faut ajouter une prime de risque. $\mathrm{Si}$, par exemple, l'utilité marginale de la richesse nominale future est positivement corrélée avec le taux d'inflation, la prime de risque est positive. Le différentiel d'intérêt est alors supérieur au taux d'inflation anticipé. Dans ce cas, en effet, l'actif réel est moins risqué que l'actif nominal, bien que son rendement nominal est incertain, car son rendement nominal est élevé (faible) lorsque l'utilité marginale de la richesse nominale est élevée (faible) c'est-à-dire lorsque le besoin d'argent est élevé (faible). Il est donc relativement plus avantageux de détenir l'actif réel. Par conséquent, l'actif nominal doit offrir un rendement supérieur à ce qui est nécessaire pour protéger l'épargne contre l'inflation de façon à compenser le risque que le besoin d'argent soit élevé (risque qui n'est pas assuré par l'actif nominal dans la mesure où son rendement nominal est le même dans tous les états de la nature).

Il est nécessaire de distinguer le comportement vis-à-vis du risque d'un individu suivant que le choix porte sur des loteries dont les gains sont des sommes d'argent ou des loteries dont les gains sont des paniers de biens. La raison est que dans un contexte d'incertitude sur le taux d'inflation une somme d'argent donnée aura une utilité variable en fonction du prix des biens de consommation. Nous allons donc devoir distinguer deux types de neutralité au risque : la neutralité nominale et la neutralité réelle suivant que les choix portent sur des loteries nominales ou réelles.

On dira qu'un individu est neutre au risque de richesse nominale si sa fonction d'utilité indirecte est linéaire par rapport à la richesse nominale. Dans ce cas, le multiplicateur de Lagrange $\lambda$ sera constant puisque, d'après le théorème de l'enveloppe, il est égal à l'utilité marginale de la richesse nominale en première période. À cause de la séparabilité de la fonction d'utilité directe, la fonction d'utilité indirecte sera identique aux deux périodes et l'utilité marginale de la richesse nominale $\left(\mathrm{U}^{\prime}\left(\mathrm{C}_{2 s}\right) / \mathrm{P}_{2 s}\right)$ en seconde période sera constante quel que soit l'état de la nature et égale à $\lambda$. De plus, la neutralité nominale n'implique pas que l'agent représentatif est victime de l'illusion monétaire. En effet, on sait que la fonction d'utilité indirecte est homogène de degré zéro par rapport aux prix et à la richesse nominale. Par conséquent, si les prix et la richesse nominale sont multipliés par deux, l'utilité de l'agent représentatif n'en sera pas modifiée.

On dira que l'individu est neutre au risque de consommation si sa fonction d'utilité directe est linéaire par rapport à la consommation auquel cas l'utilité marginale de la consommation sera constante. De plus, et cela est valable aussi bien pour la neutralité réelle que pour la neutralité nominale, l'hypothèse de neutralité de l'agent représentatif n'implique pas que tous les consommateurs sont neutres au risque. Il suffit en effet qu'un seul d'entre eux soit neutre au risque pour que la tolérance au risque du groupe soit infinie ${ }^{1}$.

1. Cf. L. Eeckhoudt et C. Gollier [1992], p. 210-214. 
Fort de ces définitions, nous sommes en mesure de déterminer deux relations de Fisher. D'une part, la relation traditionnelle lorsque l'individu est neutre au risque de richesse nominale :

$$
\frac{(1+\mathrm{R})}{(1+r)}=\frac{\mathbf{E} \tilde{\mathbf{P}}_{2}}{\mathbf{P}_{1}}
$$

D'autre part, lorsque l'individu est neutre au risque de consommation on a la relation :

$$
\frac{(1+r)}{(1+\mathrm{R})}=\frac{\mathrm{E} \tilde{\delta}_{2}}{\delta_{1}}
$$

Ces deux relations étant identiques dans le cas de prévision parfaite où se plaçait I. Fisher [1896].

Notons enfin que, dans le cas où l'individu représentatif est neutre au risque de richesse nominale, les prix d'états et les probabilités corrigées du risque sont égales à, respectivement :

$$
\begin{gathered}
q_{s}=\beta \pi_{s} \\
\hat{\pi}_{s}=\pi_{s}
\end{gathered}
$$

Dans le cas où l'individu est neutre au risque de consommation, on $a$, en revanche :

$$
\begin{gathered}
q_{s}=\beta \pi_{s} \delta_{2 s} / \delta_{1} \\
\hat{\pi}_{s}=\frac{\pi_{s} \delta_{2 s} / \delta_{1}}{\sum_{s=1}^{\mathrm{S}} \pi_{s} \delta_{2 s} / \delta_{1}}
\end{gathered}
$$

On constate ainsi un biais entre les probabilités corrigées du risque et les vraies probabilités dans le cas où l'agent est neutre au risque de consommation, alors que ce biais disparaît dans le cas où l' agent est neutre au risque de richesse nominale.

\section{RELATION DE FISHER ET NEUTRALITÉ DE L'INFLATION}

La relation (25) est souvent associée à l'idée de neutralité de l'inflation. Les anticipations inflationnistes seraient parfaitement incorporées dans le taux d'intérêt nominal de telle sorte que le taux d'intérêt réel reste constant. Il est par conséquent assez paradoxal de constater que c'est précisément lorsque (25) est vérifiée que le taux d'intérêt nominal est constant et que le taux d'intérêt réel décroît avec l'inflation anticipée. En effet, on a d'après (15) et (16), lorsque l'agent représentatif est neutre au risque de richesse nominale :

$$
\begin{gathered}
(1+\mathrm{R})=\beta^{-1} \\
(1+r)=\beta^{-1} / \mathrm{E}\left(\tilde{\mathrm{P}}_{2} / \mathrm{P}_{1}\right)
\end{gathered}
$$


En revanche, lorsque les conditions nécessaires à la réalisation de (26) sont vérifiées (neutralité au risque de consommation), l'inflation anticipée est neutre. En effet, on a, d'après (15) et (16), les relations :

$$
\begin{gathered}
(1+\mathrm{R})=\beta^{-1} / \mathrm{E}\left(\tilde{\delta}_{2} / \delta_{1}\right) \\
(1+r)=\beta^{-1}
\end{gathered}
$$

Il apparaît ainsi que la relation qui devrait être invoquée en faveur de l'hypothèse de la neutralité de l'inflation n'est pas celle qui l'est en réalité dans les modèles macro-économiques et que c'est la relation (26) qui devrait être appelée relation de Fisher.

Cependant, on n'a pas le droit de se livrer à ce type d'exercice de statique comparative puisque le taux d'inflation anticipée est une variable endogène. En revanche, le prix aléatoire de la consommation future est un paramètre exogène du modèle. Nous allons donc étudier l'influence du prix futur sur l'inflation anticipée et sur les taux d' intérêt. Il est clair qu'une telle étude ne peut être menée à bien que dans le cadre de l'ensemble des équations du modèle (13)(17) et non à partir des équations (15)-(16) uniquement.

Toute variation du prix aléatoire de la consommation future entraîne simultanément une variation de l'inflation anticipée et de son incertitude. Il est bon cependant, d'un point de vue analytique, d'étudier séparément ces deux influences. C'est pourquoi nous étudierons tout d'abord les conséquences d'une augmentation certaine du prix aléatoire futur, puis les conséquences d'une dilatation de sa distribution à espérance constante.

Supposons donc tout d'abord que le prix futur du bien de consommation augmente de $\varepsilon$ dans tous les états de la nature. Différentions le système d'équations (13)-(17) par rapport à $\varepsilon$ et évaluons le en $\varepsilon=0$. On pourra déterminer ainsi l'influence d'une augmentation parfaitement anticipée de l'inflation sur les variables du modèle et en particulier sur les taux d'intérêt.

Des équations (14) et (17), on tire la relation :

$$
\frac{d \mathbf{P}}{d \varepsilon} 1=\frac{d \lambda}{d \varepsilon}=0
$$

c'est-à-dire une augmentation certaine du prix futur est sans influence sur le prix courant de la consommation et sur l'utilité marginale de la richesse nominale. On est par conséquent parfaitement autorisé à parler d'augmentation certaine du taux de l'inflation anticipé. En tenant compte de (35), on tire des équations (13), (15) et (16)

$$
\begin{aligned}
& \mathrm{P}_{2 s} \frac{d \mathrm{C}}{d \varepsilon} 2 s=(1+r) \bar{b}+\frac{\bar{b} \mathrm{P}_{2 s}}{\overline{\mathrm{P}}_{1}} \frac{d r}{d \varepsilon}+\overline{\mathrm{B}} \frac{d \mathrm{R}}{d \varepsilon}-\mathrm{C}_{2 s} \quad s=1, \ldots, \mathrm{S} \\
& (1+\mathrm{R}) \sum_{s=1}^{\mathrm{s}} \pi_{s} \frac{\left(\mathrm{P}_{2 s} \mathrm{U}^{\prime \prime}\left(\mathrm{C}_{2 s}\right) \frac{d \mathrm{C}}{d \varepsilon} 2 s-\mathrm{U}^{\prime}\left(\mathrm{C}_{2 s}\right)\right)}{\mathrm{P}_{2 s}}+\frac{d \mathrm{R}}{d \varepsilon} \sum_{s=1}^{\mathrm{s}} \pi_{s} \frac{\mathrm{U}^{\prime}\left(\mathrm{C}_{2 s}\right)}{\mathrm{P}_{2 s}}=0 \\
& (1+r) \sum_{s=1}^{\mathrm{S}} \pi_{s} \mathrm{U}^{\prime \prime}\left(\mathrm{C}_{2 s}\right) \frac{d \mathrm{C}}{d \varepsilon} 2 s+\frac{d r}{d \varepsilon} \sum_{s=1}^{\mathrm{S}} \pi_{s} \mathrm{U}^{\prime}\left(\mathrm{C}_{2 s}\right)=0
\end{aligned}
$$


Grâce à (36) on peut réduire ce système à un système de deux équations à deux inconnues $\mathrm{dr} / \mathrm{d} \varepsilon$ et $d R / d \varepsilon$. Les solutions de ce système sont cependant trop complexes pour que l'on puisse en donner une interprétation simple. Cependant, cela est possible dans le cas où l'agent représentatif est neutre au risque. Il peut sembler a priori quelque peu surprenant que l'aversion au risque ait une influence quelconque sur les solutions de ce système. En effet, on cherche à établir l'influence d'une augmentation certaine du prix futur du bien de consommation. Cependant, cette augmentation certaine a une influence, variable suivant les états de la nature, sur la consommation future comme le montre l'équation (36).

Considérons d'abord le cas de la neutralité au risque de consommation. Des équations (37) et (38) on tire les relations :

$$
\begin{gathered}
\frac{d \log (1+\mathrm{R})}{d \varepsilon}=\frac{\sum_{s=1}^{s} \pi_{s} /\left(\mathrm{P}_{2 s}\right)^{2}}{\sum_{s=1}^{s} \pi_{s} / \mathbf{P}_{2 s}}>0 \\
\frac{d \log (1+r)}{d \varepsilon}=0
\end{gathered}
$$

L'augmentation certaine du taux de l'inflation anticipé se traduit donc, comme le prédisaient les équations (33) et (34), par une hausse du taux d'intérêt nominal ; le taux d'intérêt réel, quant à lui, n'est pas affecté par cette variation.

Dans le cas de la neutralité au risque de richesse nominale, l'utilité marginale de la richesse nominale est constante. Par conséquent, on doit avoir :

$$
\frac{\mathrm{U}^{\prime \prime}\left(\mathrm{C}_{2 s}\right)}{\mathrm{U}^{\prime}\left(\mathrm{C}_{2 s}\right)} d \mathrm{C}_{2 s}-\frac{d \mathrm{P}_{2 s}}{\mathrm{P}_{2 s}}=0 \quad s=1, \ldots, \mathrm{S}
$$

d'où l'on tire :

$$
\mathrm{U}^{\prime \prime}\left(\mathrm{C}_{2 s}\right) \frac{d \mathrm{C}}{d \varepsilon} 2 s=\frac{\mathrm{U}^{\prime}\left(\mathrm{C}_{2 s}\right)}{\mathrm{P}_{2 s}}(=\text { constante par hypothèse }) s=1, \ldots, \mathrm{S}
$$

En utilisant (42) on tire des équations (37) et (38) :

$$
\begin{gathered}
\frac{d \log (1+\mathrm{R})}{d \varepsilon}=0 \\
\frac{d \log (1+r)}{d \varepsilon}=\frac{-1}{\sum_{s=1}^{\mathrm{S}} \pi_{s} \mathrm{P}_{2 s}}<0
\end{gathered}
$$

Comme dans le cas précédent, les résultats obtenus (43)-(44) ne sont pas différents de ceux qu'on aurait obtenus à partir des équations (31)-(32). Cela tient à ce qu'une variation du prix de la consommation future est, d'après (35), sans influence sur le prix de la consommation courante. L'augmentation certaine du taux de l'inflation anticipé se traduit donc, comme le prédisaient les équations (31) et (32), par une diminution du taux d'intérêt réel ; le taux d'intérêt nominal, quant à lui, n'est pas affecté par cette variation. 
Il résulte donc de cet exercice que la relation de Fisher sous sa forme traditionnelle (25) ne saurait être associée à l'hypothèse de neutralité d'inflation puisqu'une hausse parfaitement anticipée du taux d'inflation se traduit par une diminution proportionnelle du taux d' intérêt réel. En revanche, la relation (26) est, quant à elle, parfaitement compatible avec l'hypothèse de neutralité de l'inflation puisqu'une hausse parfaitement anticipée du taux d'inflation se traduit par une augmentation proportionnelle du taux d'intérêt nominal.

Par ailleurs, on notera que bien que la relation de Fisher sous sa forme traditionnelle (25) ne corresponde pas à l'hypothèse de la neutralité de l'inflation, elle est néammoins plus réaliste que la relation (26). En effet d'après (44), le taux d'intérêt réel et le taux d'inflation anticipé sont corrélés négativement ce qui, d'après J.-P. Danthine et J.B. Donaldson [1986], constitue le fait stylisé fondamental à expliquer. Par ailleurs, toujours d'après ces auteurs, les travaux empiriques n' ont pu mettre en évidence de corrélation entre taux d'intérêt nominal et infiation, ce qui est prédit par (43) ${ }^{1}$.

Nous allons maintenant étudier l'influence de l'incertitude de l'inflation sur les taux d'intérêt. Supposons que, dans chaque état de la nature $s$, le prix aléatoire de la consommation future augmente de $\sigma\left(E\left(\tilde{\mathrm{P}}_{2}\right)-\mathrm{P}_{2 s}\right)$. Ainsi l'incertitude diminue avec l'augmentation de $\sigma^{2}$. Différentions donc le système (13)(17) par rapport à $\sigma$ et évaluons-le en $\sigma=0$. Des équations (14) et (17) on tire la relation :

$$
\frac{d \mathbf{P}}{d \sigma} 1=\frac{d \lambda}{d \sigma}=0
$$

c'est-à-dire une augmentation de l'incertitude du prix futur est sans influence sur le prix courant de la consommation et sur l'utilité marginale de la richesse nominale. On est par conséquent parfaitement autorisé à parler d'augmentation de l'incertitude du taux de l'inflation. Des autres équations du modèle, on obtient :

$$
\begin{array}{r}
\mathrm{P}_{2 s} \frac{d \mathrm{C}}{d \sigma} 2 s=(1+r) \frac{\bar{b}}{\mathrm{P}_{1}}\left(\overline{\mathrm{P}}_{2}-\mathrm{P}_{2 s}\right)+\frac{\bar{b} \mathrm{P}_{2 s} \frac{d r}{\mathrm{P}_{1}}+\overline{\mathrm{B}} \frac{d \mathrm{R}}{d \sigma}+\left(\mathrm{P}_{2 s}-\overline{\mathrm{P}}_{2}\right) \mathrm{C}_{2 s}}{s=1, \ldots, \mathrm{S}} \\
(1+\mathrm{R}) \sum_{s=1}^{s} \pi_{s} \frac{\mathrm{P}_{2 s} \mathrm{U}^{\prime \prime}\left(\mathrm{C}_{2 s}\right) \frac{d \mathrm{C}}{d \sigma} 2 s-\mathrm{U}^{\prime}\left(\mathrm{C}_{2 s}\right)\left(\overline{\mathrm{P}}_{2}-\mathrm{P}_{2 s}\right)}{\left(\mathrm{P}_{2 s}\right)^{2}}+ \\
+\frac{d \mathrm{R}}{d \sigma} \sum_{s=1}^{\mathrm{s}} \pi_{s} \frac{\mathrm{U}^{\prime}\left(\mathrm{C}_{2 s}\right)}{\mathrm{P}_{2 s}}=0
\end{array}
$$

1. Cf. note 2, p. 585.

2. Cf. S. Fischer [1972]. Une augmentation de $\sigma$ diminue la variance de $\tilde{\mathrm{P}}_{2}$ tout en conservant son espérance mathématique. La dispersion de $\tilde{\mathrm{P}}_{2}$ autour de son espérance s'en trouve réduite. En effet, lorsque $P_{2 s}$ est relativement faible (c'est-à-dire si $\mathbf{P}_{2 s}<\overline{\mathbf{P}}_{2}$ ) il est remplacé par un prix de la consommation future plus grand $\left(\hat{\mathrm{P}}_{2 s}=\mathrm{P}_{2 s}+\sigma\left(\overline{\mathrm{P}}_{2}-\mathrm{P}_{2 s}\right)\right)$ et vice versa lorsque $\mathrm{P}_{2 s}$ est relativement élevé. Une augmentation de $\sigma$ correspond donc à une contraction de la distribution du prix de la consommation future, c'est-à-dire à une diminution de l'incertitude. 


$$
(1+r) \sum_{s=1}^{\mathrm{S}} \pi_{s} \mathrm{U}^{\prime \prime}\left(\mathrm{C}_{2 s}\right) \frac{d \mathrm{C}}{d \sigma} 2 s+\frac{d r}{d \sigma} \sum_{s=1}^{\mathrm{S}} \pi_{s} \mathrm{U}^{\prime}\left(\mathrm{C}_{2 s}\right)=0
$$

Comme dans l'exercice de statique comparative précédent, il n'est pas possible, en général, de tirer des équations (46)-(48) des conclusions claires quant à l'influence de l'incertitude de l'inflation sur les taux d'intérêt. En revanche, lorsque l'individu représentatif est neutre au risque de consommation, on a :

$$
\begin{gathered}
\frac{d \log (1+\mathrm{R})}{d \sigma}=\frac{-\operatorname{Cov}\left(\tilde{\mathrm{P}}_{2},\left(1 / \tilde{\mathrm{P}}_{2}\right)^{2}\right)}{\mathrm{E}\left(1 / \tilde{\mathrm{P}}_{2}\right)}>0 \\
\frac{d \log (1+r)}{d \sigma}=0
\end{gathered}
$$

Ainsi une augmentation de l'incertitude (une diminution de $\sigma$ ) n'a, dans le cas où (26) est vérifiée, aucune influence sur le taux d'intérêt réel et, plus surprenant, une influence négative sur le taux d'intérêt nominal.

Dans nos économies modernes, on a observé qu'un taux d'inflation moyen plus élevé est associé à une plus grande incertitude de l'inflation ${ }^{1}$. Par conséquent, même si (26) est valable, l'hypothèse de neutralité de l'inflation ne sera pas vérifiée puisqu'une augmentation de l'inflation sera accompagnée d'une augmentation moins que proportionnelle du taux d'intérêt nominal $R$. En effet, l'augmentation du taux d'intérêt nominal due à l'augmentation du taux d'inflation espéré est compensée partiellement par l'effet négatif dâ à l'augmentation de l'incertitude. (41):

Dans le cas de la neutralité au risque de richesse nominale, on a, d'après

$$
\mathrm{U}^{\prime \prime}\left(\mathrm{C}_{2 s}\right) \frac{d \mathrm{C}}{d \sigma} 2 s=\frac{\mathrm{U}^{\prime}\left(\mathrm{C}_{2 s}\right)}{\mathrm{P}_{2 s}}\left(\mathrm{E}\left(\tilde{\mathrm{P}}_{2}\right)-\mathrm{P}_{2 s}\right) \quad s=1, \ldots, \mathrm{S}
$$

Cette relation nous permet de déduire des équations (47) et (48) :

$$
\begin{aligned}
& \frac{d \log (1+\mathrm{R})}{d \sigma}=0 \\
& \frac{d \log (1+r)}{d \sigma}=0
\end{aligned}
$$

Ainsi dans le cas où (25) est vérifié, les équations (52) et (53) montrent que l'incertitude de l'inflation n'a pas d'influence sur les taux d'intérêt. Par conséquent, compte tenu de la corrélation de fait entre incertitude et inflation anticipée, on peut dire que, dans un monde où la relation de Fisher (25) serait respectée, l'inflation devrait être corrélée négativement avec le taux d'intérêt réel et il ne devrait pas y avoir de corrélation significative entre l'inflation anticipée et le taux d'intérêt nominal. Comme il est reconnu que c'est assez exactement ce qui prévaut dans les faits, nous sommes en droit de conclure que la relation de Fisher sous sa forme traditionnelle (25) est une hypothèse qu'on ne saurait écartée. Toutefois, cette hypothèse n'implique pas la neutralité de l'inflation.

1. Cf. A. Okun [1981], p. 211-212. 


\section{CONCLUSION}

Les modèles précédents ont mis en évidence l'importance du rôle de l'aversion nominale par rapport à l'aversion réelle (aversion au risque de consommation). En effet, dans une économie où les contrats sont stipulés en unité monétaire, les primes de risque doivent leur existence à l'aversion au risque de richesse nominale des agents économiques et non à leur aversion au risque de consommation. Ces deux notions d'aversion au risque ont cependant des liens qu'il serait intéressant d'étudier de façon approfondie.

Phus fondamentalement, l'étude des modèles nous révèle la nature profonde du risque d'un actif. Un actif n'est pas risqué simplement parce que son rendement est incertain, où même parce qu'il donne lieu à des pertes en capital, mais parce que son rendement est corrélé négativement avec l'utilité marginale de la richesse $^{1}$. Dans ce cas, en effet, le rendement est élevé (bas) lorsque son utilité est petite (grande). Le véritable risque que fait courir un actif, c'est de rapporter beaucoup quand on en a pas besoin et de rapporter peu alors qu'on en a besoin. Pour inciter un agent économique rationnel à investir dans un tel actif, il faudra lui offrir une prime.

Il serait intéressant d'intégrer à la structure des modèles en plus des opérations de consommation déja prises en compte, les opérations de production. Nous pourrions ainsi étudier l'influence du cycle économique sur les taux d'intérêt. De plus la prise en compte de l'État nous permettrait d'étudier l'influence de la politique économique sur ces mêmes variables.

On ne peut pas cependant enrichir d'éléments nouveaux la structure du modèle de bases sans précaution. En effet, certains d'entre eux ${ }^{2}$ peuvent supprimer l'équivalence entre équilibre compétitif et optimalité. Or c'est cette équivalence qui nous permet de considérer le programme du consommateur maximisant son utilité comme un programme de maximisation de l'utilité collective par le planificateur. Cela nous a permis d'interpréter les prix d' équilibre obtenus par cette méthode ${ }^{3}$ comme ceux qui prévaudrait à l'équilibre d'une économie de concurrence parfaite. Dans le cas où l'équivalence classique de la théorie du bien-être n'est pas vérifiée, les prix qui sont obtenus sont tels que les agents économiques ne sont pas incités à échanger entre eux (c'est-à-dire ce sont des prix d'autarcie). Il est évident que, dans cette situation, les enseignements que l'on peut tirer de cette méthode ont une importance limitée.

1. Nominale si le rendement est nominal ou réelle si le rendement est réel.

2. Comme les coûts de transaction ; cf. F. Hahn [1988].

3. Procéder directement à partir de l'équilibre des offres et des demandes compliquerait les calculs de façon prohibitive ; cf. R Lucas [1987]. 


\section{REFÉRENCES BIBLIOGRAPHIQUES}

BenNinga Simon, Protopapadakis Aris [1983], «Real and Nominal Interest Rates Under Uncertainty : the Fisher Theorem and the Term Structure », Journal of Political Economy, p. 856-867.

DANTHINE Jean-Pierre, Donaldson John B. [1986], «Inflation and Asset Prices in an Exchange Economy ", Econometrica, p. 585-605.

Demange Gabrielle, Rochet Jean-Charles [1992], Méthodes mathématiques de la Finance, Paris, Economica.

EecKHOUDT Louis, GollIER Christian [1992], Les risques financiers, Paris, Ediscience International.

FISHER Irving [1896], Appreciation and Interest, Publications of the AEA, New York, Macmillan.

FISCHER Stanley [1972], « Slutsky Equations for Assets », Econometrica.

FrENKEL Jacob A., RAZIN Assaf [1980], « Stochastic Prices and Tests of Efficiency of Foreign Exchange Markets », Economics Letters, p. 165-170.

HAHN Frank [1988], « On Monetary Theory », Economic Journal, p. 957-973.

HANSSON Ingemar, STUART Charles [1986], « The Fisher Hypothesis and International Capital Markets », Joumal of Political Economy, p. 1330-1337.

LuCAS Robert E., Jr. [1987], Models of Business Cycles, Yrjö Jahnsson Fondation, Oxford, Basil Blackwell.

MASSE Pierre [1948], «La notion d'espérance marginale, la théorie générale de J.M. Keynes et le problème de l'intérêt », Revue d'économie politique, p. 88-132.

OKuN Arthur [1981], Prices and Quantities, Oxford, Basil Blackwell, 367 p.

RoLI. Richard, SoLNIK Bruno [1979], «On some Parity Conditions Encountered Frequently in International Economics ", Journal of Macroeconomics, p. 267-283.

SARGENT Thomas J. [1987], Dynamic Macroconomic Theory, Cambridge, Harvard University Press.

SVENSON Lars E. O. [1985], «Money and Asset Prices in a Cash-in-Advance economy », Journal of Political Economy, p. 919-944.

VARIAN Hal R. [1984], Microeconomic Analysis, $2^{\mathfrak{e}}$ éd., New York, Norton.

VARIAN Hal R. [1988], "Le principe d'arbitrage en économie financière », Annales d'économie et de statistique, p. 348-361. 